\title{
Converged Fixed and Radio-over-Fiber Link Employing Optical Envelope Detection and Optically Injected DFB Laser
}

Prince, Kamau; Tafur Monroy, Idelfonso

Published in:

OFC/NFOEC 2008 - 2008 Conference on Optical Fiber Communication/National Fiber Optic Engineers Conference

Link to article, DOI:

10.1109/OFC.2008.4528447

Publication date:

2008

Document Version

Publisher's PDF, also known as Version of record

Link back to DTU Orbit

Citation (APA):

Prince, K., \& Tafur Monroy, I. (2008). Converged Fixed and Radio-over-Fiber Link Employing Optical Envelope Detection and Optically Injected DFB Laser. OFC/NFOEC 2008 - 2008 Conference on Optical Fiber

Communication/National Fiber Optic Engineers Conference, OThD7. https://doi.org/10.1109/OFC.2008.4528447

\section{General rights}

Copyright and moral rights for the publications made accessible in the public portal are retained by the authors and/or other copyright owners and it is a condition of accessing publications that users recognise and abide by the legal requirements associated with these rights.

- Users may download and print one copy of any publication from the public portal for the purpose of private study or research.

- You may not further distribute the material or use it for any profit-making activity or commercial gain

- You may freely distribute the URL identifying the publication in the public portal 


\title{
Converged fixed and radio-over-fiber link employing optical envelope detection and optically injected DFB laser
}

\author{
K. Prince ${ }^{1}$ and I. Tafur Monroy ${ }^{1}$ \\ 1. Department of Communications Optics \& Materials, COM•DTU, Ørsteds Plads, bldg 343, DK-2800 Kgs. Lyngby, Denmark \\ kpr@com.dtu.dk
}

\begin{abstract}
We present $2.5 \mathrm{~Gb} / \mathrm{s}$ all-optical RF signal down-conversion from $20 \mathrm{GHz}$ by exploiting an optically-injected DFB. We demonstrate converged transmission of this signal with a baseband PON over a common $70 \mathrm{~km}$ fiber link. (C)2008 Optical Society of America

OCIS codes: (060.0060) Fiber optics and optical communications; (060.5625) Radio frequency photonics
\end{abstract}

\section{Introduction}

Next-generation broadband networks will be characterized by their ability to deliver high-speed services directly to end users over larger coverage areas while meeting or exceeding current levels of reliability and availability. Recent advances towards meeting this challenge include the deployment of fiber-to-the-customer-premises (FTTCP) optical networks, in which optical infrastructure directly links customer sites with the central office (CO). One of the drivers for FTTCP is the demand anticipated for bandwidth-intensive applications including high-definition TV (HDTV), for example. Parallel demands on wireless networks for increased flexibility and access speeds to mobile TV, video and broadband multimedia services will also accelerate the rate at which core networking speeds must be increased. The introduction of optical fiber network equipment at the customer premises will allow the extension of high-speed wireless network access by the introduction of suitable wireless access point (WAP) equipment.

Seamless integration of traffic destined for both fixed and wireless access networks onto the same infrastructure for FTTCP, while supporting the correct protocols required for each destination network, remains as an interesting challenge to network designers. An additional requirement is the development and integration of low-complexity WAPs that support the high data transfer rates typically implemented in passive optical network (PON) systems.

We consider a PON in which signals from the $\mathrm{CO}$ are optically broadcasted to each optical network unit (ONU) over a common optical feeder fiber via a passive remote node (RN) optical splitting/combining point. A time division multiplexing (TDM) access protocol ensures collision-free upstream connectivity from $\mathrm{ONU}$ to $\mathrm{CO}$. We assume data transmission at $2.5 \mathrm{~Gb} / \mathrm{s}$, which is typical of current PON implementations [1].

This paper proposes and experimentally demonstrates a proof-of-principle PON system that simultaneously supports radio-over-fiber and fixed upstream traffic. This $2.5 \mathrm{~Gb} / \mathrm{s}$ system facilitates baseband data transmission while simultaneously implementing frequency down-conversion and detection of a data stream ASK modulated onto a $20 \mathrm{GHz}$ carrier. The WAP performs all-optical half-wave rectification without the use of radio-frequency oscillators: the RN uses an optically injected DFB laser to perform envelope detection for the half-wave rectified signal from the WAP. The resulting system implements baseband signal transport between RN and CO.

2. Fixed and radio-over-fiber converged link with all-optical envelope detection

A possible converged fixed and radio-over-fiber link is presented in Figure 1a. The wireless signals received at the WAP is used to drive a properly biased electro-absorption modulator (EAM) so that half-wave rectification is achieved [2]; see Figure 1b. This half-wave rectified optical signal is then sent to the RN. With the implementation of a suitable TDM scheduling scheme, collision-free signal arrival (from ONU and WAP nodes) is assumed at the $\mathrm{RN}$. As in a conventional PON, the signals are then multiplexed onto a single datastream by using a passive optical

(a)

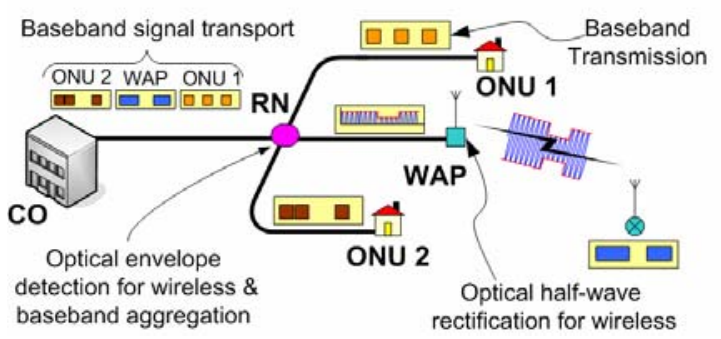

(b)

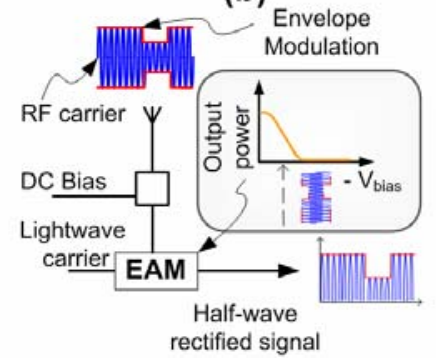

(c)

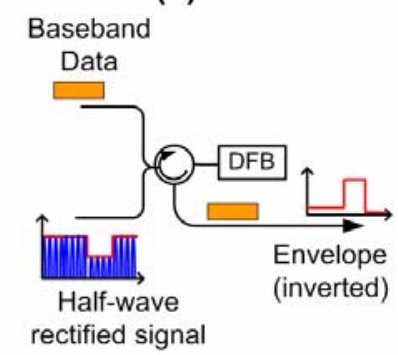

Figure 1a: Diagram of upstream channel for a converged fixed and radio-over-fiber link over passive optical network architecture (PON). CO: central office. RN: remote node. WAP: wireless access point. ONU: optical network unit (b) Diagram of optical half-wave rectification using an electro-absorption modulator. (c) Remote node employing an external optical injected DFB laser performing envelope detection 
combiner. The multiplexed datastream is injected into a DFB laser biased above lasing threshold so that the digital transmission levels either allow or suppress DFB laser output. Due to the speed of the gain suppression dynamics, the DFB response is fast enough to follow the baseband signals and the ASK envelope, but unable to track the high frequency radio-frequency carrier (see Figure 1c). The proposed RN in Figure 1a therefore performs all-optical envelope detection. Data transmission between RN and CO therefore occurs only at baseband frequencies regardless of the source node type. This greatly reduces the requirements for dispersion management and marks a significant improvement over the more stringent requirements imposed by conventional radio-over-fiber links. We additionally believe that the injected laser may be further optimized to enhance optical signal extinction and amplification and thus expand the reach and/or scalability of a PON network.

Our proposed system considers a single $\mathrm{CO}$ and $\mathrm{RN}$ separated by $50 \mathrm{~km}$; the distance between the WAP/ONU and the RN is $20 \mathrm{~km}$. We assume that the short hop is implemented with $20 \mathrm{~km}$ single mode fiber (SMF) and evaluate the performance of the longer-distance uplink using alternately SMF (with dispersion of $17 \mathrm{ps} / \mathrm{nm} / \mathrm{km}$ ) or nonzero dispersion-shifted fiber (NZDSF) with $5 \mathrm{ps} / \mathrm{nm} / \mathrm{km}$ dispersion. A pair of Mach-Zehnder modulators (MZM) were used to produce the $2.5 \mathrm{Gbit} / \mathrm{s}$ ASK signal with $20 \mathrm{GHz}$ carrier; this simulated an optically half-wave rectified WAP output. The baseband $2.5 \mathrm{~Gb} / \mathrm{s}$ ONU signal was simulated by disabling the $20 \mathrm{GHz}$ modulator. The system diagram is presented as Figure 2.

The laser used was a commercially available multiple-quantum well (MQW) InGaAs distributed feedback (DFB), coax packaged device, without output optical isolation. It was operated with no temperature or wavelength stabilization circuitry. The central emitting wavelength $\lambda_{C}$ was observed to lie around $1551 \mathrm{~nm}$. The wavelength of the external injected light was set at $1553 \mathrm{~nm}$ to coincide with the closest non-lasing mode of the DFB at a wavelength higher than $\lambda_{\mathrm{C}}$. For all the reported measurements, the DFB bias current was maintained at $21.10 \mathrm{~mA}$ and the average optical power injected into the DFB was kept at $-7.5 \mathrm{dBm}$.

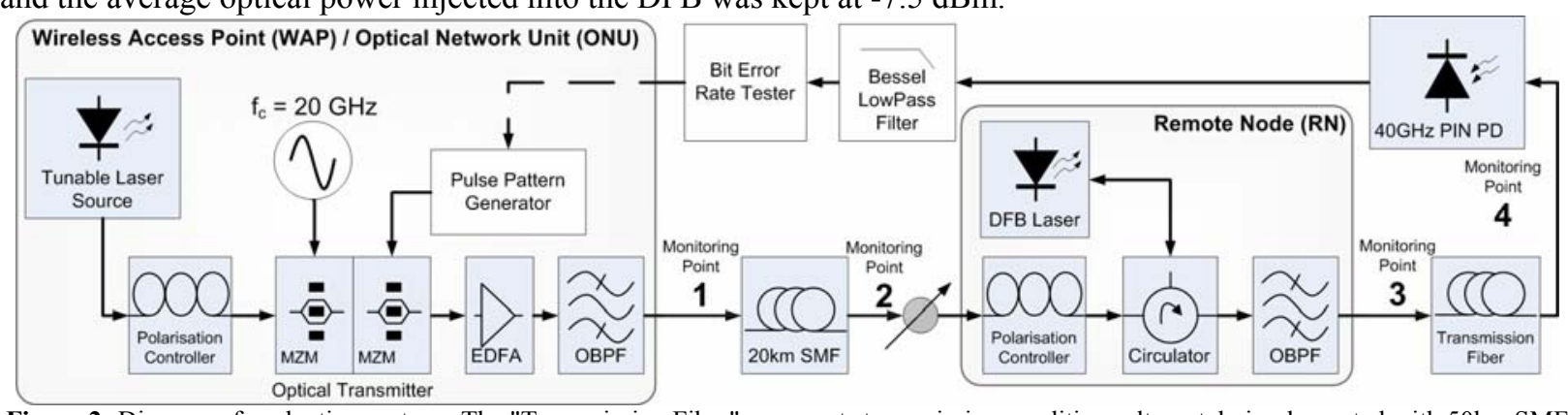

Figure 2: Diagram of evaluation system. The "Transmission Fiber" represents transmission conditions alternately implemented with 50km SMF \& 50km NZDSF. OBPF: Optical bandpass filter; MZM: Mach-Zehnder modulator.

\section{Results}

Sample waveforms obtained at different points of the proposed system are presented in Figure 3 below. Subfigures $3 \mathrm{a}$ and $3 \mathrm{~b}$ indicate the observations at the DFB output when the RN is connected directly to the wireless access point WAP/ONU of Figure 1: this is known as the back-to-back scenario. 3a presents the result when the injected signal is the ASK modulated signal on the $20 \mathrm{GHz}$ carrier (carrier on), and $3 \mathrm{~b}$ presents the results obtained when the injected signal is the baseband $2.5 \mathrm{GHz}$ signal (baseband). Subfigure 3c shows the input signal at measuring point 1 (MP1), a zoomed view indicates the presence of digital ASK modulation. 3d indicates the results obtained at MP3; after the DFB. Subfigures 3e and 3g indicate the results obtained at MP4 when the SMF and NZDSF are respectively used as the transmission fiber: these waveforms were obtained after photodetection with a $40 \mathrm{GHz}$ PIN photodiode. Figures $3 \mathrm{f}$ and $3 \mathrm{~h}$ present the results at MP4: after electrical low-pass filtering with a 1.8 GHz Bessel filter. Results are again presented for SMF and NZDSF fiber transmission case.

We observe that the output of the DFB is able to successfully follow the envelope of the input signal when this input is at baseband or is ASK-modulated $20 \mathrm{GHz}$ carrier. The DFB output was also observed to exhibit overshoot and high-frequency transients typical of relaxation oscillations associated with laser-switching applications. These transients affected the opening of the eye of the optical signal at MP3 and MP4; eye opening was later restored by low-pass filtering after photodetection.

Receiver sensitivity was assessed at various points, with the results presented in Figure 4. In this figure, the solid icons indicate performance with injection of the ASK modulated $20 \mathrm{GHz}$ signal from the WAP, while partlyshaded icons indicate performance with the ONU baseband input. Results are also presented for the back-to-back test scenario. These results indicate that RN performance very closely follows that of the input signal stream for both baseband and $20 \mathrm{GHz}$ ASK input. System performance was affected by dispersion along the transmission fiber. 


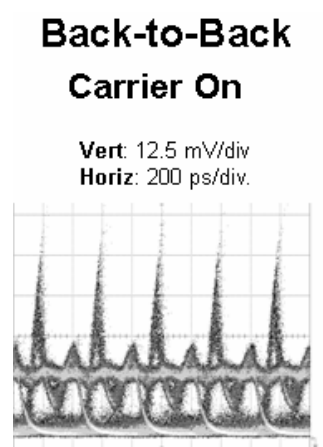

(a)

Baseband

Vert: $12.5 \mathrm{mV} / \mathrm{div}$ Horiz: $200 \mathrm{ps} / \mathrm{di}$.

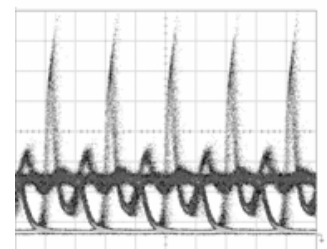

(b)

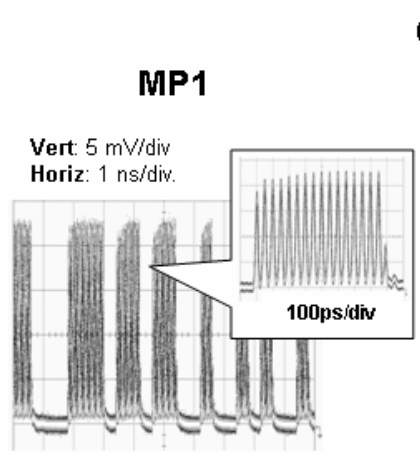

(c)

\section{MP3}

Vert: $10 \mathrm{mV} / \mathrm{div}$

Horiz: $200 \mathrm{ps} / \mathrm{div}$.

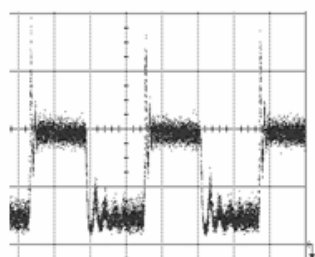

(d)

\section{Carrier On}

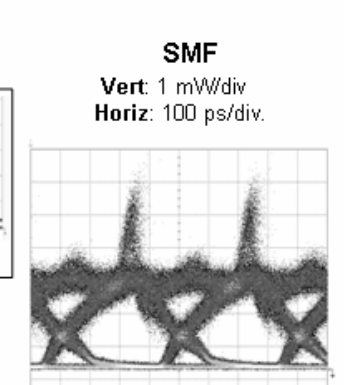

(e)

SMF, LPF

Vert: $74.7 \mathrm{mV} / \mathrm{div}$

Horiz: 100 ps/div.

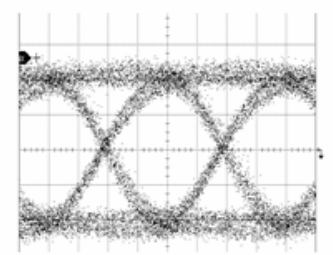

(f)
MP4

NZDSF

Vert $700 \mu \mathrm{W} / \mathrm{div}$ Horiz: $100 \mathrm{ps} / \mathrm{div}$

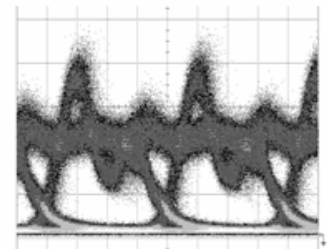

(g)

Vert $49,6 \mathrm{mV} / \mathrm{div}$ Horiz: $100 \mathrm{ps} / \mathrm{div}$.

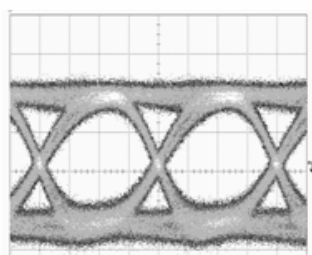

(h)

Figure 3: Signal waveforms obtained at various points through the system. Subfigures a and $\mathbf{b}$ indicate the observations at the DFB output when connected directly to the WAP/ONU of Figure 1: this is the best-case for transmission, with carrier on and baseband waveform injection. Subfigures $\mathbf{c}-\mathbf{h}$ indicate the transmission with carrier on input at TP1.Sunbfigures $\mathbf{e} \& \mathbf{g}$ indicate the signal at TP4; the resulting output of a 1.8 $\mathrm{GHz}$ bandlimited electrical filter is presented as subfigures $\mathbf{f} \& \mathbf{h}$. These indicate transmission via SMF and NZDSF.

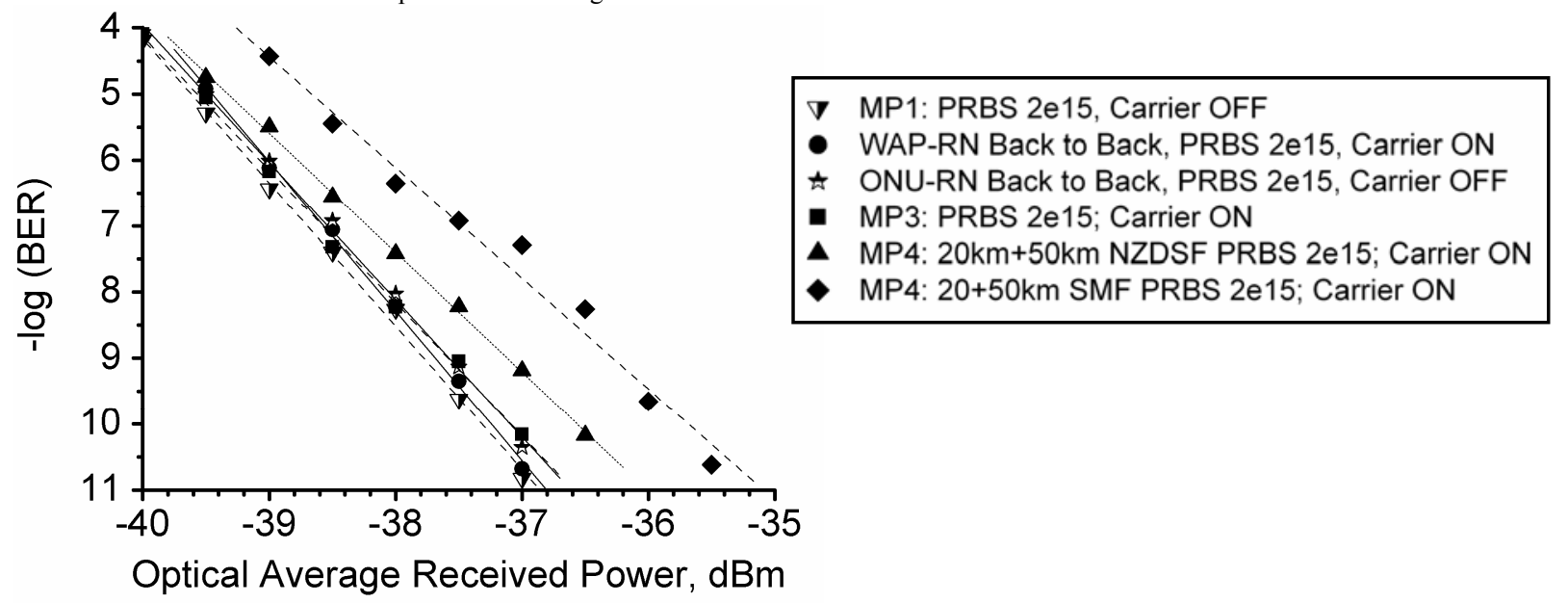

Figure 4: Measured BER curves at various points through the system. Solid icons indicate $2.5 \mathrm{~Gb} / \mathrm{s}$ ASK modulated input (with $20 \mathrm{GHz}$ carrier): partly-shaded icons indicate injection of a baseband $2.5 \mathrm{~Gb} / \mathrm{s}$ waveform. Results also indicate the back to back performance.

\section{Conclusion}

We presented successful converged signal transport of optical signals formatted for both baseband ONU as well as radio-over-fiber applications via a $2.5 \mathrm{~Gb} / \mathrm{s}$ PON with $70 \mathrm{~km}$ between end-nodes and the CO. Successful all-optical envelope detection for wireless signals has been achieved with an external optically injected DFB laser with support for baseband signals. BER measurements were presented: these show that good system performance was maintained after the DFB laser. These results indicate that the power penalty of the receiver sensitivity at BER of $10^{-9}$ was just below $1.5 \mathrm{~dB}$ with $50 \mathrm{~km}$ SMF, and was below $0.7 \mathrm{~dB}$ for $50 \mathrm{~km}$ when NZDSF was used.

Further investigation will be done to better understand the behaviors of the novel PON implementation. This research will establish the operating limits and examine the potential for optimized implementations of this system.

\section{References}

[1] T. Koonen, "Fiber to the home/fiber to the premises: what, where, and when?" Proc IEEE, vol. 94, pp. 911-934, 2006.

[2] I. Tafur Monroy, J. Seoane and P. Jeppesen, "All-Optical Envelope Detection for Wireless Photonic Communication," in 33rd European Conference and Exhibition on Optical Communication (ECOC2007), 2007. 